\title{
L'énergie au point de vue juridique (Etat de la législation actuelle)
}

\author{
Par Paul Bougault, Avocal à la Cour d'Appel de Lyon.
}

\begin{abstract}
(SUITE)
$\underline{(S U I E)}$

L'auteur continue l'article commencé au numéro précédent, dans lequel il avait indiqué les règles principales applicables aux permissions de voirie; il entreprend aujourd'hui l'étude de la concession simple, c'est-á-dire de la concession ordinaire telle qu'elle se présente quotidiennement, sans déclaration d'utilité publique.
\end{abstract}

\section{SFCTION TROISIEME : CONCESSION SIMPLE}

Paragraphe $1^{\mathrm{er}}$. - Règles particulières à ce genre de concession

Principes généraux. - La distribution d'énergie, telle qu'elle est organisée par la loi du 15 juin 1906 (titre IV) constitue évidemment une concession de travaux publics, puisque aux termes de tous les cahiers types officiellement adoptés par divers décrets, l'entrepreneur accepte la charge d'établir, ou au moins d'entretenir, les ouvrages nécessaires à la distribution, et, à l'expiration même du contrat, il les abandonne gratuitement à l'autorité ('oncédante, les résultats de l'exploitation ayant dû le rémunérer de ses avances.

Mlais si l'obligation d'établir et d'entretenir des ouvrages necupe, dans le contrat, une place tellement prépondérante qu'elle le fait classer parmi les concessions de travaux publics, le concessionmaire n'cn est pas moins le gérant d'un service public, dont le but est de fournir le courant èlectrique à certaines collectivités ; à ce dernier point de vue, et sans s'arrêter aux différentes catégories entre lesquelles se répartissent les voies occupées, les articles 6 et 7 de la loi fixent les autorités compétentes pour donner la concession ; si la distribution ne dépasse pas la limite d'une eommune ou d'un ensemble de communes syndiquées, le Maire, après délibération $d u$ Conseil municipal, ou le Président du Syndicat après avis favorable du Comité, signe le contrat qui est ensuite soumis à l'approbation préfectorale; dans tous les autres cas, l'Etat seul intervient, représenté par le Préfet pour une dislribution s’étendant sur un seul département à des communes non syndiquées, ou par le Ministre dans le cas contraire.

Soumise à toutes les régles générales en matière de concession de travaux publics, la distribution d'energie trouve certaines règles qui lui sont spéciales dans divers articles de la loi du 15 juin 1906.

Règles spéciales. - L'article 10 constate le droit pour le concessionnaire d'exécuter sur les voies publiques et leurs dépendances les travaux nécessaires à l'établissement et à l'entretien des onvrages, en se conformant aux conditions du cahier des charges, des règlements de voirie, et des règlements d'administration publique prévus par la loi. L'Administration ne saurait done lui refuser, sans motif légitime, les permissions qui lui sont nécessaires; mais si des modifications sont exigées l'Administration a le droit de les imposer, sauf indemnité dont le règlement est confié aux 'Tribunaux compétents, à moins qu'clle soil dêfinic par le cahier des charges; il est à remarquer que le cahier lype, dans son article deuxième, indique que les changements sernt supportés sans indemnité, quand ils seront requis pour un motif de sécurité publique ou dans l'intérêt de la voirie. La concession est donc soumise sur ce point aux règles ci-dessus prescrites pour la permission.

Le silence gardé par cet article au sujet des tiers s'explique par l'application normale dés principes gẹnéraux qui suffisent à résoudre les questions de voisinage entre un concessionnaire et les propriétaires riverains, d'après la formule suivante : le concessionnaire en occupant le sol, comme il en a le droit, peut devenir la cause de certains inconvénients ; mais, sous peine de dommagesintérêts, il ne doit jamais dépasser une mesure qui est laissée à l'appréciation des Tribunaux administratifs ; il en est ainsi, alors même que la concession de distribution serait déclarée d'utilité publique ; car la compétence du Juge de paix, au sujet des servitudes spéciales prévues par l'article 12 de la loi du 15 juin 1906, est limitée à la fixation de l'indemnité due pour leur établissement, les principes de la compétence administrative restant intacts pour les inconvénients du voisinage (Cons. Etal, 2 avril 1919, Société des Tramways de Calais contre Dagniaull, Rec. Cons. Et. page 339). Le Conseil d'Etat a estimé dans certains cas, qu'un filet métallique, placé à une trop grande proximité des fenêtres d'une maison, est une gêne pour les locataires et diminue le revenu de l'immeuble. (Cons. Etat 9 juillet 1913, Société des Tramways électriques de Lille, Dal. 1917, 7. 3. 23) et, dans d'autres cas, que des vibrations et du bruit ne constituaient pas une gène suffisante pour légitimer l'allocation d'une indemnité (Cons. Etat, 6 décembre 1912, Cle du Métropolitain. Dal. 1916. 3. 71).

L'article 9 interdit à l'autorité concédante d'exiger du concessionnaire une charge pécuniaire autre que des tarifs de faveur pour l'Etat et la commune, et des redevances prévues au règlements ( $\$ 7$ de l'article 18). La loi a vouli éviter que la concession envisagée dégénèrât dans ce mode conträctuel qui, sous le nom de régie intéressée, permet au pouvoir concédant de se créer des ressources pécuniaires en partageant avec le concessionnaire, soit dès le début, soit dès qu'elles atteignent un certain chiffre, les recettes obtenues ou une fraction dés bénéfices. (Sur la différence entre la régie intéressée et la cóncession proprement dite de travaux publics, voir note sous l'arrèt du 6 avril 1895, Deshayes contre ville de Lorient, Dal. 1896, 3. 33).

L'article 8 édicte le principe que le concessionnaire ne pourra s'opposer à la concurrence qui lui serait faite par un autre concessionnaire; mais les conditions imposées au second ne doivent pas être plus avantageuses que celles imposées au premier; l'article déclare même que sous la réserve d'égalité dans les conditions, le concessionnaire initial ne peut s'opposer it la concurrence d'un 
permissionnaire: mais, en prati(ue, cette égalité de conditions ne peut ètre obtenue entre deux régimes si différents.

A cette faculté donnée à la concurrence, l'article 8 ne préroit qu'une exception ; dans le cas d'une concession municipale d'énergie, on sait que cette concession peut ètre domnée : poue tous usages, ce qui comprend, d'une part, la lumière, et, dautrr part, tous les emplois différents (force motrice, chaleur, elc...), ou bien pour la lumière seulement, ou enfin pour les usages autres que la lumière. Quand un cahier réglemente la distribulion de la lumière, soit seule, soit réunie à la distribution du courant pour d'autres usages, il peut accorder le bénéfices de l'exclusivité, en faveur de léclairage seulement, à la condition qu'elle soit expressement stipulée à l'article 2 du cahier des charges et soit strictement conforme aux prescriptions de l'article 8 de la loi du 15 juin 1906 ; d'où il résulte : $1^{\circ}$ que son extension à la force motrice entrânerait la nullité de la délibération du Conseil Innicipal qui l'aurait promise au concessionnaire : (Conseil d'Etat, 12 juillet 1918); que même légalement limité à l'éclairage le privilège ne saurait empècher le distributeur de force motrice (concessiomnaire ou permissionnaire) de foumir l'éclairage accessoire à cette force, dans les locaux où l'énergie est utilisée (article 8 de la loi) ; $3^{\circ}$ qu'à partir du jour mème, en vertu d’un avenant formel, le privilège exclusif promis en ce qui concerne Ies particuliers a été supprimé, la commune n'engage pas sa responsabilité en octroyant des autorisations de voirie, ou en laissant l'Etat occuper, pour domer de la lumière au service des Postes, un bâtiment communal, la commune n'élant plus engagée qu'en ce qui concerne les services municipaux (Conseil Etal. 27 juillet $1923, \mathrm{C}^{\text {ie }}$ du Gaz Lebon et $\mathrm{C}^{\text {ie }}$ contre Ville de Quimper. La Cour de Cassation par arrêt du 20 octobre 1913, Bardot contre Société des Moulins de Gray, constate cette innovation de la loi de 1906 permettant à une commune de créer un privilège qui porte sur toute l'étendue de son territoire, c'est-à-dire sur des voies même ne dépendant pas du domaine communal.

La loi du 15 juin 1906 ayant consacré une différence entre le courant-lumière et le courant-force en ce qui concerne le privilìge, de nombreuses difficultés sont survenues, car les tarifs, plus clevés pour la lumière que pour la force motrice, onl incité les consommateurs à convertir en éclairage le courant réservé à d'autres usages : les concessiomaires se sont vivement opposés à cette pratique : déjà, avant la loi, il avait été jugé par le Conseil d'Etat (arrèt du 23 décembre 1904, Société des Forces Motrices du Rhòne, Dal. 1906. 3. 66) que si un cahier des charges interdil au concessionnaire de consacrer à la lumière, sans une autorisation formelle, l'énergie qu'il produil, cette interdiction répétée dans la police s'impose au consommateur; depuis la loi, il a été jugé : $1^{\circ}$ par la Cour d'appel de Grenoble (arrêt du 16 décembre 1917, non publié, Société de Force et Lumière contre Arbel) que le distributeur concessionnaire peut, en vertu de l'article $8^{7}$ de la loi, s'opposer à ce que le courant-force soit utilisé sous forme de lumière, dans un local qui, bien que dépendant d'une usine à laquelle le courant est fourni, est consacré aux réunions du Conseil d'Administration et au logement des Administrateurs en tournée ; $2^{\circ}$ par la Cour d'Appel de Paris (arrêt du 25 avril 1917, $\mathrm{C}^{10}$ Continentale Edison contre Société Universelle Cinéma, non publié), et sur pourvoi par la Chambre des Requêtes (4 mars 1919, Rev. des Conc., année 1919, page 152), que le courant ulilisé par la lanterne cinématographique, bien qu'il soit nécessaire de lui faire subir certaines transformations, est un courant-lumière, puisqu'il rend lumineux un écran obscur par lui-même.

Création des cahiers-types. - Enfin, d'après l'article 6 de la loi, loute concession est donnée en conformité d'un cahier týpe dont le texte, approuvé par décret, le Conseil d'Etat entendu, comprend deux parties : l'une imprimée en caractères ordinaires qui est abligatoire et à laquelle on ne saurait apporter deśs modificalions dans un décret d'approbation délibéré en Conseil d'Etat (article 7, \$5), lautre en caractères italiques qui est facullative en ce sens que les parties s'entendent pour la rayer ou la laisser subsister : elles fixent aussi les conditions de fourniture, de tarifs, les détails du fonctionnement, cte..., qui varient avee chaque cas particulier.

Les trois types de cahiers des charges officiellement approuvés sont destinés : le premier à la concession donnée par une commune ou un Syndicat de communes (décret du 17 mai 1908, modifié le 28 juin 1921); le deuxième, à la concession afférente à plusienrs communes non syndiquées du mème département (dẻcret du 20 anût 1908 , modifié le 28 juin 1921) ; le troisiène à la concession domnée par l'Etat dans le but de fournir le courant à des services publies (décret du 30 novembre 1909, modifié le 28 juin 1921).

Cette énumération est loin d'ètre limitative, en ce sens que d'autres $(y) e s$ de cahiers pourront ètre adoplés; nolamment, il en sera ainsi pour l'opération dite de transport de courant qui sera rendue particulièrement fréquente grâce à la loi du 19 juillet 1922.

Parngraphe 2e. - Le cahier type d'une distribution communale.

Cerlains articles de ce cahier ne présentent aucune particularite, notamment tous ceux qui relèvent du domaine technique ou ceux qui sont empruntés à la législation bien connue des chemins de fer. D'autres, au contraire, déjà commentés par la jurisprudence, ont une importance très grande pour la rédaction du cahier.

Examen des articles spéciaux. - L'article $1^{\text {er }}$ permet de préciser, après l'usage auquel le courant est destiné, quelle sera l'étendue territoriale de la concession; on principe, elle comprentoute la surface de la commune : si l'on veut soustraire certaines zones, il est nécessaire d'inscrire au contrat des réserves formelles; il en est de mème au sujet du maintien de certaines installations antérieures; d'un arrêt rendu le 20 janvier 1922 (Rev. des Cone. année 1922, page 253) par le Conseil d'Elat sur une concession avec privilège exclusif existant antérieurement au cahicr type, il résulle que la responsabilité de la commune ne saurail ètre engagée, même en l'absence de réserves, à raison d'installations existantes au moment de la signature de la convention ; le même principe doit ĉtre appliqué sous le régime du cahier lype.

L'article 5, d'après sa première rédaction dans le cahier type du 17 mai 1908, a admis : $1^{\circ}$ que les usines de production, à. moins d'être formellement énumérées au contrat, ne seraient pas englobees dans la concession; $2^{\circ}$ que le réseau comprendrail au moins un poste central qui ferait partie de la concession et l'alimenterait : $3^{\circ}$ que le concessionnaire ferait à ses frais l'établissement de tout le réseau ; $4^{\circ}$ que la commune pourrait lui donner à bail, moyennant le paiement d'un loyer, le réseau dont elle serait propriétaire ; cette dernière éventualité se réalisera de plus en plus fréquemment, à mesure que, par rachat ou extinction des concessions actuelles, les communes acquerront des réseatix.

Mais, par une adjonction très heureuse, le décret du 28 juin 1921 permet à la commune d'accorder, pour la construction du réseall, une subvention forfaitaire ou proportionnelle aux dépenses ef limitée aux 4/5 de celles-ci ; cette disposition a été inspirée par l'impossibilité d'établir les réseaux, dans les conditions actuelles, et d'utiliser les subventions que les communes rurales pourront obtenir. La commune peul exiger en compensation de la subvention une redevance spéciale, calculée d'après le nombre de kilowatts-heure distribués ou d'après les bénéfices réalisés.

L'artìcle $B$ définit le réseau en exigeant du concessionnaire 
If depôl d'un plan qui en dome la consistance ; à ce lilre, il a une importance considérable pour délimiter le parcours de la distribution, car, sur ce parcours, d'apres l'arlicle 13, le concessionnaire est tenu de fournir le comant aux consommateurs, dans un délai généralement court à compler de la demande el, en vertu de l'arlicle 14, il est tenu d'élablir les lignes de jonction necessiares au service des consommaleurs qui assurent pendant un temps domné une réserve minima.

L'article 11 vise les Larifs maxima que le concessionnaire est toujours en droil d'abaisser el qu'il peul ensuile reprendre, sans (quit l'accomplissement d'aucune formalité puisse être exigé par la commune (Cons. d'Et. 18 février 1921, Ville de Méru, Rec. Cons. Et. page 192) ; mais, s'il les abaisse pour certains abomés, il devra faire profiter de celle amélioration tous ceux qui se trourent dans les mêne conditions de puissance et d'horaire.

La commune et le concessionnaire sont libres d'adopter les larifs relatifs à la fourniture au compteur et à la fournilure à forfait ; mais, le décret du 28 juin 1921, à titre d'indication, a introduit dans les conditions facultatives la notion de "l'Index "cononique " destinée à faire varier le prix de la consommation en suivant les modilications du prix de revient ; les données essentielles de la combinaison peuvent se résumer ainsi : on appelle "index "un prix de charbon calculé d'après le prix réel et le salaire horaire de la main-d oeuvre ; le tarif de base est fixé pour le kilowatt-heure d'après un index égal à 130 ; à chaque trimestre, le Ministere des Travaux publics fait publier pour toutes les régions l'index réel ; le cahier des charges prévoit qu'à chaque franc d'écart entre l'index initial el lindex réel, le prix du kilowall-heure variera en plus ou en moins dun certain nombre de millimes.

Quand il survient une difficulté au sujet des tarifs, el d'une façon générale, d'une obligation vraie ou prétendue du concessionnaire, il faut examiner au point de rue de la compétence quelles sont les parties litigantes : $1^{\circ}$ si le débat existe entre la Municipalité et lo concessionnaire, la compétence du Conseil de Prélecture est indisculable ; à cette juridiction seule, il appartient de préciser les obligations du concessionnaire vis-à-vis de la commune, à la condition de ne le condamner qu'à des dommagesintérèts s'il y a lieu, sans fixer d'astreinte pour l'exécution d'un fail précis (Cons. d'Etat 17 novembre 1922, Sté Electricité d"Anjou contre commune de Chalonnes, Rev. des Conc. 1923, p. 145) ; la rounmune est également sans qualité pour demander le remboursement par la Compagnie aux abonmés des sommes que ceux-ci onl indûnent versées, notamment à la suite d'une réduction de l'éclairage, soit pour en réclamer le versement à la Caisse Muni(ipale, a titre de dommages-intérèts (Cons. Elal, 20 janvier 1896, Ville de Saint-Elienne, Dal. 1897. 3. 9) ; 20 si le débat existe entre 111. consommateur et la commune, sur le sens el l'exécution du trailé, le Tribunal de droit commun doit affirmer sa compétence; mais il lui est défendu d'interpréter une tause ambiguë (Ch. Civ.' Cas. 28 juin 1922, Préaubert, Ste Nantaise d'Eelairage, Rev. des conc, 1923, p. 139), doit être casse larrèt qui, après avoir affirme qu il se bornat a appliquer des clatuses clatres et préeises, a domi, en réalité, nue interprotation à une elatase que la Cour de Cassation déclare obseure. (Ch. Civ., 28 juin 1923, Fevdel et lales contre Andrienx, Gaz, des Trib., 10 aont 1923 ).

Dans le cas do renvoi, le Conseil de Préfecture sans retenir laffaire devant lui, doit donner simplement l'interprétation qui hit est demandée (Cons. d'Elat, 24 décembre 1897, Cie du Caz do Bordeaux, Ville de Bordeaux, Dal, 1899, 3. 34). Mais, tant que lo Conseil d'titat n'a pas stalué, l'arrêté du Conseil de Préfecture contre lequel il a été formé un pourvoi, ne doit pas être considérí comme définilif par le Tribunal de droit commun, le principe de l'exẻcution immédiale des arêtés cle Préfecture n'étant pas applicable relativement à une interprétation sollicitée par la
Juridiction Civile: les juges peuvent toutefois régler entre ahonnés el concessionnaires le paiement de la consommation ; el agissant ainsi, ils se bornent à respecter la clatuse d'une police qui a stipulé le paiement malgré tout débat judiciaire (Cour de Rentnes, 18 décembre 1922, Société des A teliers de Brelagne, Societé Vanlaise d'Electricité, Bul. des Ls. Elec, 1923, page 11).

L'article 12 détermine le prix de revient que le concossiontnaire devra accorder aux services publics de l'Etal, des depurtements, établissements publies, et certaines associations syndicales ; il conlient aussi les stipulations spéciales à la fourniture dénergic que la commune entend se faire réserver, avec les tarifs applicables à ces fournitures, sans que la commune soit dans l'obligation d'exiger pour soil eclairage public, le tarif le plus bas. (Conseil d'Elat, 12 mars 1915 ).

L'arlicle 15, en sinspirant de la jurisprudence constante du Conseil d'Etat, altribue au concessionnaire la propriéé du branchement extérieur, c'est-à-dire etabli sur ou sous la voie publique, tout en obligeant le consommateur à rembourser d'après un tarif convemu, le coût de cet établissement. Au contraire, les branchements intérieurs (colonnes montantes et dérivations) sont établis par les soins des propriétaires des immeubles.

En conséquence, le branchement extérieur faisant partie de la concession constitue un travail public qui rend l'entrepreneur responsable des accidents causés par ce travail (Cons. Etat, 30 juillet 1914), dame Zuiglen, Ville de Paris), car, en thèse générale, même quand aucune faute n'est révélée à la charge du concessionnaire, il est responsable et doit réparer les conséquences des inconvéuients causés aux tiers. (Cons. Et., 21 mai 1919, Garay contre Cie du Gaz de Lyon, Rec. Cons. Etat, page 445). Bien que le branchement reste une dépendance de la concession, il est juste et légal que le cahier des charges mette le remboursement des frais à la charge de l'abonné : statuant sur un cahier des charges qui était muet sur la question du remboursement, la Chambre des Requêtes (arrêt du 8 mars 1922, Cie du Gaz de Condé-sur-Noireau contre Ruault (Rev. des Conc., 1922, p. 347) a adinis qu'aucun principe n'interdit au concessionnaire de réclamer une rémunération pour ce travail de raccordement, sauf à ne pas l'exiger, en dehors d'une stipulation formelle du contrat, sous forme d'abonmement, ce qui constítuerait une augmentation sur le prix prévu pour le gaz.

Les articles 16 et 17 indiquent les conditions de la foumiture des compteurs et fixent le droil du concessionnaire de vérifier le fonctionnement : il a été jugé par la Cour d'Appel d'Orléans (arrêt du 7 février 1922, S... contre Cie d'Electricité, Bul. des Us. Elec. 1922, page 37) que l'abonné qui possède un compteur d'une puissance trop forte pour qu'une comsommation modeste puisse ètre enregistrée, ne peut pas se plaindre de lenlèvement chu compteur ; les distributeurs ont le droit d'exiger qu'un appareil de mesure soit en rapport avec la consommation qu'il doit enregistrer.

L'arficle is domne au concessionnaire le droil do ne pas fommir le courant arant qu'une police ait été signée; la jurisprudence, avant mème le cahier des charges, avait déjà statué en ce sens (Voir C. de Paris, 15 février 1906, Sté Electrique de St-Chéron c. Viau. Dal. 1906. 5, p. 52). Le distributeur, par ce moyen, fait engager sa clientèle à respecter les clauses du cahier des charges ; ainsi disparait cette question de fait, tranchée en des sens différents suivant les circonstances, en l'absence de police : le consommateur était-il soumis aux clauses du cahier des charges, ou pouvait-il se dire ignorant des conditions générales? Une Société qui distribue du gaz à diverses communes et le reçoit d'une autre Société qui, comme concessionnàxe d'une Tille, est tènue de le lui fournir d'après les tarifs d'un cahier des charges, n'est pas recevable $\hat{a}$ dire qu'elle ignore leur modification (Ch. des Req., 4 mai 1921, Sté Intercommuaale du Gaz contre Cie du Gaz de 
Bordeaux, Dal. 1922. 1. 10); il a ètè au contraire jugé qu'un particulier contre lequel le concessiomaire aux lieu et place d'une police, ne peut alléguer que des quillances de paiement, est recevable à ne pas se dire lié d'après le cahier des charges (Ch. des Re(., 20 juillet 1920, de Rodellec contre Briand. Dal. 1922. 1. 40) ; de mème, après avoir fait sans police une fourniture provisoire, à titre d'essai, un concessionnaire ne saurait, par une application directe du cahier des charges, exiger du consommateur le minimum de recette que larticle 13 lui permet de réclamer d'un abonné (Ch. Civ. Cas., 23 février 1923, Grandin contre Sociélé Electrique d'Anjou. Dal. 1923, Chron. p. 11).

L'article 21 détermine la durée de la concession ; le maximum est de 40 ans ; quelle que soit la durée, l'échéance du terme oblige le concessionnaire à remettre à la commune les installations en bou état d'entretien, à moins que celle-ci se soit réservé le droit d'en exiger l'enlèvement (art. 22).

L'article 25 est relatif à la déchéance que le Ministre des Tràvaux publics a seul le droit de prononcer malgré le caractire essentiellement municipal du contrat et "sauf recours au Conseil
d'Etal par la voie contentieuse ". Le Conseil d'lital a jugé le 10 août 1923 (Affaire Chazerans contre commune de St-Firgean. Dal. 1923. Chron. p. 34), que ces termes n'enlèvent pas au Conseil de Préfecture jugeant en premier ressort, le droil de statuer sur les difficultés survenues entre concédant et concessionnaire, et que ce dernier ne peut se servir de la voie d'un recours pour excès de pouvoir contre l'arrèté ministériel pour le faire annuler. Inversement, puisque le Ministre seul est en droil de prononcer la déchéance, le Conseil de Préfecture ne peul se déclarer compétent sur la demande que la commune lui adresse directement a cet effet; il en est ainsi alors mème que le distributeur possède deux qualités : d'abord, celle de concessionnaire de la distribution d'énergie, en vertu d'un contrat conforme au cahier lype; ensuite celle de concessionnaire gazier en vertu d'un contrat qui ne contient aucune réserve du droit pour l'autorité supérieure de prononcer la déchéance. Le Conseil de Préfecture doit donc juger sur la résiliation qui lui a été juridiquement demandée au sujet du contrat de distribution de gaz (Cons. Etat, 26 janvier 1923, Ville de Toulon contre Société d'Eclairage de Toulon, Rev. des Conc. 1923, page 124 et Dal. 1923, Chron. p. 22).

\section{DOCUMENTATION La Centrale de Ritom.}

Par E. GARNIER, ancien élève de l'Ecole Polytechnique, Ingénieur à la Société Alsacienne et Lorraine d'Ëlectricité.

Depuis la fin de l'année 1920 fonctionne, en Suisse, la Centrale de Rilom destinée à l'électrification des chemins de fer et particulièrement, en liaison avec l'usine d'A msieg, à la traction sur la ligne qui va d'Erstfeld, près de Lucerne, à Bellinzona dans le Tessin, en englobant tout le tunnel du Gothard. La construction de cette centrale a donné lieut à des éludes et à des recherches fort intéressantes, dont nous allons dire quelqu's mots en utilisant pour cela la série d'articles parus depuis mai dernier dans la Schweizerische Bauzeitung, sous la signature de M. Eggenberger, ingénieur-adjoint à l'ingénieur en chef chargé de l'électrification des chemins de fer suisses. M. Eggenberger ne s'est occupé que des travaux hydrauliques eux-mêmes.

La Centrale de Ritom utilise la chute de la rivière de Foss, depuis le lac de Ritom jusqu'à l'embouchure de la rivière dans le Tessin. Pour utiliser la plus grande capacité possible du lac,

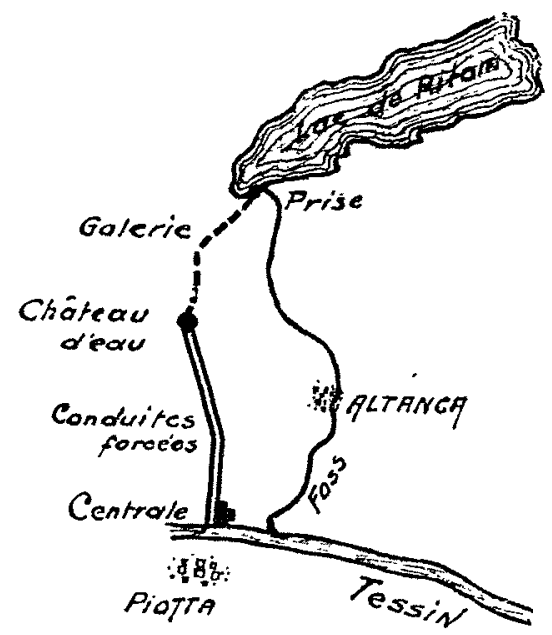

Fig. 1.

la prise d'eau fut exécutéè̀ 30 mètres de profondeur. Le bassin versant de la chute comporte $23,1 \mathrm{~km}^{2} . \Lambda$ l'aide de mesures failes depuis 1906 par le service hydrographique, on a pu établir la courbe des débits, ce qui a donné :

\section{Débit d'étiage}

$0,23 \mathrm{~m}^{3} / \mathrm{sec}$, correspondant à $10,2 \mathrm{l} / \mathrm{sec}$. par $\mathrm{km}^{2}$.

Débit des crues

$15,5 \mathrm{~m}^{3} / \mathrm{sec}$., correspondant à $669,5 \mathrm{l} / \mathrm{sec}$. par $\mathrm{km}^{2}$.

\section{Débit minima annuel}

$0,81 \mathrm{~m}^{3} / \mathrm{sec}$., correspondant à $35 \mathrm{l} / \mathrm{sec}$. par $\mathrm{km}^{2}$.

Débit moyen annuel.

$1,00 \mathrm{~m}^{3} / \mathrm{sec}$, correspondant à 43 l/sec. par $\mathrm{km}^{2}$.

Lad chute brute est de 828 mètres ce qui donne envirou 800 mìtres de chute nelte moyenne, d'où une puissance moyemne conttinue de 8.000 chevaux. Pour augmenter la capacité du lac, on décida la construction d'un barrage relevant le niveau stipéricur de 7 mètres.

Le premier problème qui se posa fut la question de l'hydrogène sulfuré qu'à partir d'une profondeur de 13 mètres contenail en grande quantité jusqu’à 28 milligrammes par litre l'eau du lac, ce qui empêchait toute possibilité de vie animale ou végétale. Il existe cependant des poissons dans le lac, mais seulement à la partie supérieure. On pouvait craindre deux choses : c'est que par suite de l'abaissement du niveau, tous les poissons du lac ne soient détruits par l'hydrogène sulfuré et, d'autre part, que 